\title{
Capnocytophaga ochracea
}

National Cancer Institute

\section{Source}

National Cancer Institute. Capnocytophaga ochracea. NCI Thesaurus. Code C86241.

A species of facultatively anaerobic, Gram-negative, fusiform, filamentous bacilli assigned to the phylum Bacteroidetes. This species is motile, non-spore forming, oxidase, catalase, and indole negative, does not produce hydrogen sulfide and does not hydrolyze gelatin, or starch, but does hydrolyze esculin. C. ochracea is a member of the normal gingival flora but can become pathogenic causing sepsis especially in immunocompromised individuals. 